\title{
Diffusion anomaly and dynamic transitions in the Bell-Lavis water model
}

Cite as: J. Chem. Phys. 133, 104904 (2010); https://doi.org/10.1063/1.3479001

Submitted: 02 June 2010 . Accepted: 23 July 2010 . Published Online: 14 September 2010

Marcia M. Szortyka, Carlos E. Fiore, Vera B. Henriques, and Marcia C. Barbosa

ARTICLES YOU MAY BE INTERESTED IN

Liquid polymorphism, order-disorder transitions and anomalous behavior: A Monte Carlo study of the Bell-Lavis model for water

The Journal of Chemical Physics 131, 164506 (2009); https://doi.org/10.1063/1.3253297

Exploiting a semi-analytic approach to study first order phase transitions

The Journal of Chemical Physics 138, 014105 (2013); https://doi.org/10.1063/1.4772809

Lattice model for water-solute mixtures

The Journal of Chemical Physics 145, 144501 (2016); https://doi.org/10.1063/1.4964396

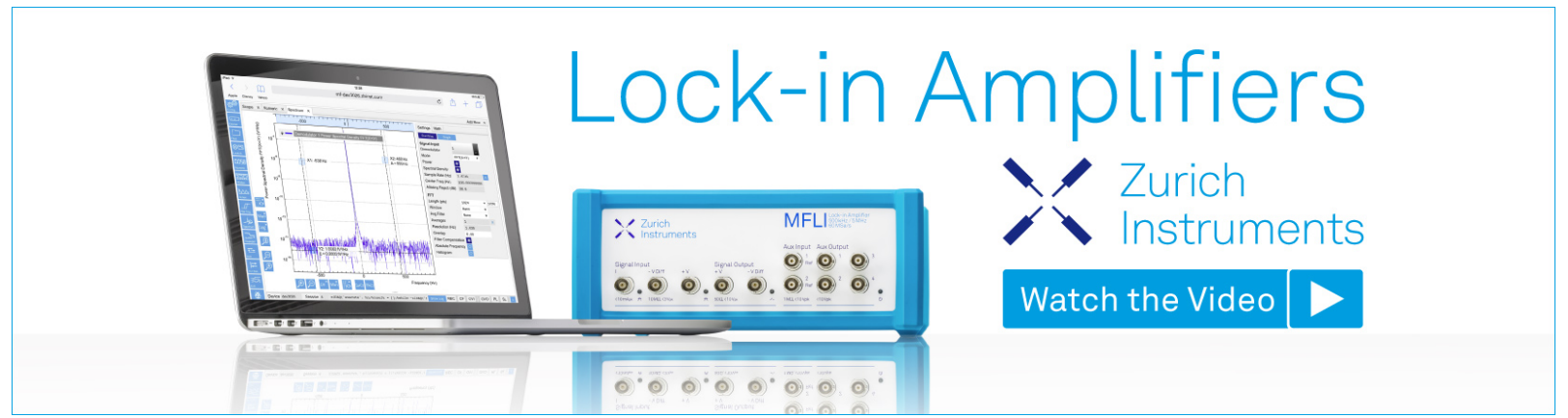

J. Chem. Phys. 133, 104904 (2010); https://doi.org/10.1063/1.3479001 


\title{
Diffusion anomaly and dynamic transitions in the Bell-Lavis water model
}

\author{
Marcia M. Szortyka, ${ }^{1, a)}$ Carlos E. Fiore, ${ }^{2, b)}$ Vera B. Henriques, ${ }^{3, c)}$ and Marcia C. Barbosa ${ }^{4, d)}$ \\ ${ }^{1}$ Departamento de Física, Universidade Federal de Santa Catarina, Caixa Postal 476, \\ 88010-970, Florianópolis, SC, Brazil \\ ${ }^{2}$ Departamento de Física, Universidade Federal do Paraná, Caixa Postal 19044, \\ 81531 Curitiba, PR, Brazil \\ ${ }^{3}$ Instituto de Física, Universidade de São Paulo, Caixa Postal 66318, 05315970, São Paulo, SP, Brazil \\ ${ }^{4}$ Instituto de Física, Universidade Federal do Rio Grande do Sul, Caixa Postal 15051, \\ 91501-970, Porto Alegre, RS, Brazil
}

(Received 2 June 2010; accepted 23 July 2010; published online 14 September 2010)

\begin{abstract}
In this paper we investigate the dynamic properties of the minimal Bell-Lavis (BL) water model and their relation to the thermodynamic anomalies. The BL model is defined on a triangular lattice in which water molecules are represented by particles with three symmetric bonding arms interacting through van der Waals and hydrogen bonds. We have studied the model diffusivity in different regions of the phase diagram through Monte Carlo simulations. Our results show that the model displays a region of anomalous diffusion which lies inside the region of anomalous density, englobed by the line of temperatures of maximum density. Further, we have found that the diffusivity undergoes a dynamic transition which may be classified as fragile-to-strong transition at the critical line only at low pressures. At higher densities, no dynamic transition is seen on crossing the critical line. Thus evidence from this study is that relation of dynamic transitions to criticality may be discarded. (ㅇ 2010 American Institute of Physics. [doi:10.1063/1.3479001]
\end{abstract}

\section{INTRODUCTION}

Water is the most familiar substance in nature and nonetheless a satisfactory understanding of its properties is still lacking. Many of its properties are regarded as anomalous when compared with those of other substances. ${ }^{1}$ Its most well-known peculiar property is probably the density anomaly, ${ }^{2}$ which increases with temperature for a range of pressures. In addition, different response functions such as specific heat, isothermal compressibility, and thermal expansion coefficient also display peculiar behaviors.

Besides thermodynamic anomalies, water also exhibits dynamic anomalies, seen in both experiments ${ }^{2}$ and in simulations. ${ }^{3}$ In usual fluids, diffusivity increases with decreasing density, since mobility is enhanced in a less dense medium. However, in the case of liquid water, a range of pressures exists for which diffusivity exhibits nonmonotonic behavior with density, and both minima and a maxima in the diffusion coefficient may be found.

It has been proposed a few years ago that these anomalies would be related to the second critical point at the end of a first order coexistence line between two liquid phases, the low-density liquid (LDL) and the high-density liquid (HDL) phases. ${ }^{4}$ This critical point, discovered through computer simulations, might be located in the supercooled region beyond the line of homogeneous nucleation and is thus inacessible experimentally. This hypothesis has been supported by

\footnotetext{
${ }^{a)}$ Electronic mail: szortyka@gmail.com.

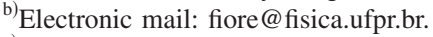

${ }^{c)}$ Electronic mail: vhenriques@if.usp.br.

${ }^{d)}$ Electronic mail: marcia.barbosa@ufrgs.br.
}

indirect experimental results. ${ }^{5}$ In spite of the limit of $235 \mathrm{~K}$ below which water cannot be found in the liquid phase without crystallization, two amorphous phases were observed at much lower temperatures. ${ }^{6}$ There is some evidence, even if not definitive, of the presence of the two liquid phases. ${ }^{7-9}$

Recently, experimental results in nanoscale hydrophilic pores show a crossover from fragile to strong diffusivity as temperature is lowered, in the supercooled region, at constant pressure. ${ }^{10-12}$ The concept of fragility, introduced by Angell, ${ }^{13}$ classifies the liquids as strong or fragile, whether the diffusion coefficient displays Arrhenius or non-Arrhenius behavior, respectively. In order to give further support to the hypothesis of a critical point at the end of the coexistence line between the two liquid phases, it was suggested that this crossover from a fragile to strong regime in water would signal the presence of criticality. In particular, it was proposed that the fragile-to-strong transition observed in water is associated with crossing the Widom line, the analytic continuation of the coexistence line. The main idea behind this assumption is that as the system approaches the critical point two structurally different liquids start to form on each side of the Widom line. On crossing the Widom line no thermodynamic transition is observed but a dynamic transition is present.

Is the fragile-to-strong transition associated to the presence of criticality in general? In order to address this question, a number of models which display criticality were investigated as to the presence of fragile-to-strong transitions. ${ }^{14-16}$ These studies have shown that on crossing the critical line, fragile-to-strong, strong-to-strong, or even fragile-to-fragile transitions could be observed, depending on the specific structure of the phases separated by the critical 
line. In the particular case of the associated lattice gas model (ALG), which presents two critical lines, two kinds of dynamic transitions are also present. The critical line separating the fluid from the low density liquid phase, at lower pressures, could be associated to a fragile-to-strong transition, whereas the critical line separating the high density fluid from the HDL phase, at higher pressures, was associated to a strong-to-strong dynamic transition. ${ }^{15}$ In both cases, the dynamic transition is of the same kind along the whole critical line. Thus, the dynamic transition upon traversing the critical line has been understood to be a result of the change of the structure of the liquid, similarly to the interpretation given to the dynamic transition seen in the case of the crossing of the Widom line. Thus a logical question arises: Is the type of the dynamic transition linked with the universality class of the critical line? or does it only depend on the nature of the phases related to the dynamic transition?

In this paper, we test these ideas on a very simple model that exhibits a single critical line separating two fluids. If the universality class of the critical line and the class of the dynamic transition are associated, we would expect the model to display dynamic transitions of one class only. We investigate the diffusion properties of the Bell-Lavis (BL) water model, ${ }^{17}$ the only two-dimensional icelike orientational model known to us which does not require an energy penalty in order to present a density anomaly. It is a triangular lattice gas model in which water molecules are represented by particles with three symmetric bonding arms interacting through van der Waals and hydrogenlike bonds. It is probably the simplest orientational model that reproduces waterlike anomalies. Our study will focus on three questions: Are dynamic anomalies and dynamic transitions verified in a minimal model? If present, how are they related to thermodynamic anomalies? Are dynamic transitions related to criticality?

This paper is organized as follows: In Sec. II the model is described and its phase diagram is reviewed; in Sec. III the simulation results for the model dynamic anomalies and dynamic transitions are presented; Sec. IV summarizes our conclusions.

\section{THE BL MODEL AND PHASE DIAGRAM}

The BL model is a two-dimensional system in which molecules are located on a triangular lattice and are represented by two kinds of variable, in order to represent occupational and orientational states. The occupational variables $\sigma_{i}$ assume the value $\sigma_{i}=0$, if the site is empty, and $\sigma_{i}=1$, if the site is occupied by a molecule. The orientational variables $\tau_{i^{i}}^{i j}$ are introduced to represent the possibility of bonding between molecules. Each molecule has six arms, separated by $120^{\circ}$, three of them inert, with $\tau_{i}^{i j}=0$, while the other three are the bonding arms, with $\tau_{i}^{i j}=1$. The two possible orientations $A$ and $B$ for the molecule are illustrated in Fig. 1.

Two neighbor molecules interact via van der Waals and hydrogen bonding. The model energy is described by the following effective Hamiltonian, in the grand-canonical ensemble

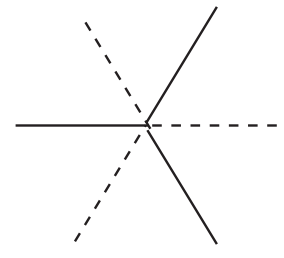

A

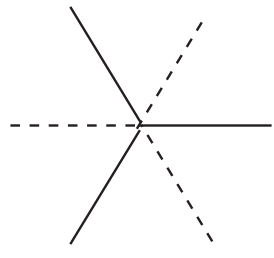

B
FIG. 1. Two possible particle orientation configurations. Solid lines are the bonding arms while dashed lines are nonbonding arms.

$$
\mathcal{H}=-\sum_{(i, j)} \sigma_{i} \sigma_{j}\left(\epsilon_{\mathrm{hb}} \tau_{i}^{i j} \tau_{j}^{i j}+\epsilon_{\mathrm{vdw}}\right)-\mu \sum_{i} \sigma_{i}
$$

where $\epsilon_{\mathrm{hb}}$ and $\epsilon_{\mathrm{vdw}}$ are the strength of hydrogen bond (hb) and van der Waals (vdW) interaction energies, respectively, and $\mu$ is the chemical potential.

In analogy to other two length scale interaction lattice models ${ }^{18-21}$ this model is expected to exhibit a region of density anomalies in its phase diagram. The phase diagram of this model was investigated for different values of the bonding strength, with different approaches: under a mean-field approach, ${ }^{17,22,23}$ with renormalization group techniques ${ }^{24,25}$ and very recently, through detailed numerical simulations. ${ }^{26}$ In this paper, we restrict our analysis to two values of the bonding strength parameter, $\zeta \equiv \epsilon_{\mathrm{vdw}} / \epsilon_{\mathrm{hb}}$, respectively, $\zeta=1 / 10$ and $\zeta=1 / 4$. These two parameter values are interesting because in both cases the system exhibits two liquid phases. However, for $\zeta=1 / 10$, the critical line ends at a tricritical point, while for $\zeta=1 / 4$ it does end at a critical end point.

The chemical potential $\bar{\mu}$ versus temperature $\bar{T}$ model phase diagram is shown in Figs. 2(a) and 2(b), for $\zeta=1 / 10$ and $\zeta=1 / 4$, respectively. Reduced units for temperature and chemical potential are defined as

$$
\bar{T}=\frac{T}{\epsilon_{\mathrm{hb}}} \quad \text { and } \quad \bar{\mu}=\frac{\mu}{\epsilon_{\mathrm{hb}}} .
$$

For both $\zeta=1 / 10$ and $\zeta=1 / 4$, the system displays three different phases. For low chemical potential, the system is constrained in the gas phase, with density $\rho \approx 0$. For intermediate values of the chemical potential, the system is in the LDL phase. For high chemical potentials, the system exhibits a HDL phase. The LDL and HDL phases are separated by a critical line, which has been identified as an order-disorder transition. ${ }^{26}$ Typical configurations for the zero temperature LDL and HDL configurations are illustrated in Fig. 3.

The phase transition between gas and LDL phases is first-order for both values of $\zeta .^{26}$ For this transition, the order-parameter is associated to density $\rho=n / V$, where $n$ is the number of occupied sites while $V=L^{2}$ is the number of sites. At zero temperature, the two phases coexist, with $\rho \approx 0$ for the gas, and $\rho \approx 2 / 3$ for the LDL. For higher bonding strength, $\zeta=1 / 10$, the coexistence line ends at a tricritical point, whereas for lower bonding strength, $\zeta=1 / 4$, it ends at a critical point. The HDL-LDL critical line ends at the coexistence line, thus yielding coexistence between the HDL and the gas phases. The phase transition between LDL-HDL 


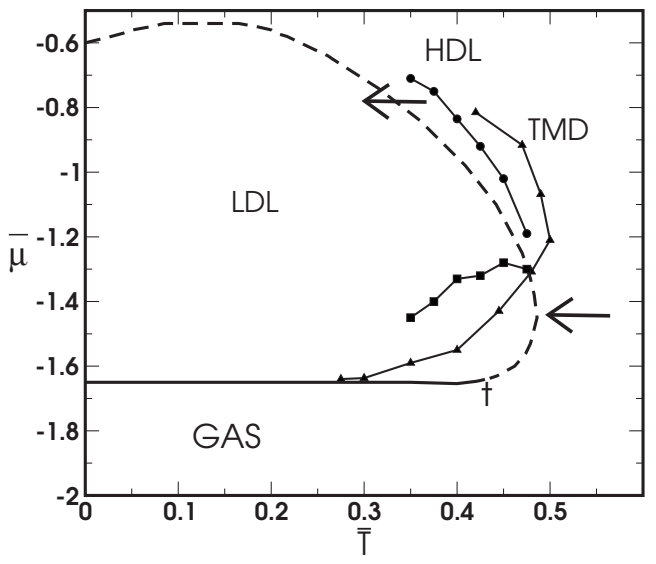

(a) $\zeta=1 / 10$.

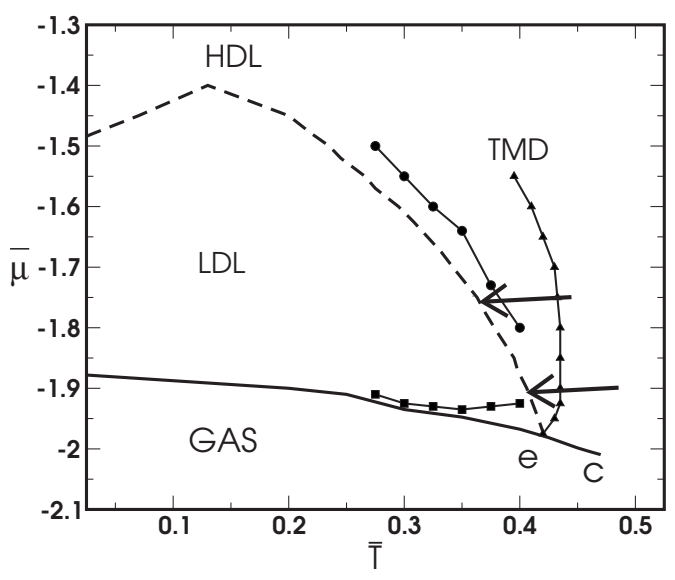

(b) $\zeta=1 / 4$.

FIG. 2. Reduced chemical potential vs reduced temperature phase diagram for (a) $\zeta=1 / 10$ and (b) $\zeta=1 / 4$. The solid line is a first order transition line between the gas and the LDL phases. The dashed line is a second order transition line between the LDL and the HDL phases. For $\zeta=1 / 10$ point $t$ is a tricritical point and for $\zeta=1 / 4$ points $e$ and $c$ correspond to end-critical point and critical point, respectively. Triangles are points of density maxima and the continuous line represents the TMD line. Circles and squares are diffusivity maxima and minima loci, respectively.

phases is second-order for both value of $\zeta$ and has been associated to an orientational order-disorder transition. ${ }^{26}$

In both cases of smaller and larger bonding strengths, the system displays a region of anomalous thermodynamic behavior. For $\zeta=1 / 4$, the line of temperatures of maximum density (TMD) is located inside the HDL phase. For $\zeta=1 / 10$, it crosses the LDL phase, for lower pressures, and migrates to the HDL phase, for high pressures.

\section{DIFFUSIVITY AND DYNAMIC TRANSITIONS}

We have studied diffusivity for the BL model over its phase diagram through Monte Carlo simulations. The numerical algorithm for studying mobility is described as follows: (i) the system is equilibrated with fixed chemical potential (or density) and fixed temperature; (ii) an occupied site $i$ and its neighbor $j$ are chosen randomly; (iii) if the neighbor site $j$ is empty, the molecule moves to the empty site and the difference between the final and the initial energy $\Delta E$ is computed; (iv) if $\Delta E<0$, the movement is accepted, otherwise the movement is accepted with a probability $\exp \left(\Delta E / k_{B} \bar{T}\right)$. A Monte Carlo step is defined through the number of trials of movement for every particle. After repeating this algorithm $n t$ times, where $n$ is the number of molecules in the lattice, the diffusion coefficient is evaluated according to Einstein's equation

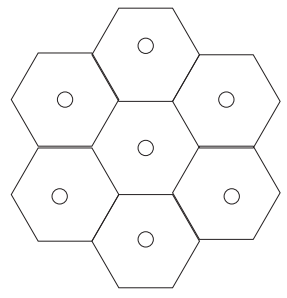

LDL

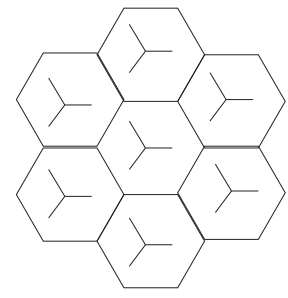

HDL
FIG. 3. Typical bond configurations for the LDL and the HDL phases at $\bar{T}=0$.

$$
\bar{D}=\lim _{t \rightarrow \infty} \frac{\left\langle\Delta r(t)^{2}\right\rangle}{4 t},
$$

where $\left\langle\Delta r(t)^{2}\right\rangle=\left\langle(r(t)-r(0))^{2}\right\rangle$ is the mean square displacement per particle and time is measured in Monte Carlo steps.

Our data have been obtained for lattice size $L=18$ under periodic boundary conditions.

\section{A. Diffusion anomaly}

In normal liquids, the diffusion coefficient grows as the density decreases. However, in anomalous liquids, the diffusivity decreases from a maximum at $\rho_{D_{\max }}$ to a minimum at $\rho_{D_{\min }}$, as the density is decreased. For densities outside this region the diffusion behaves as described above, i. e, as a normal liquid.

In order to investigate the existence of this anomaly for the BL model, the diffusion coefficient was computed as a function of density, for fixed temperatures, for both $\zeta=1 / 10$ and $\zeta=1 / 4$. The results are shown in Figs. 4(a) and $4(\mathrm{~b})$.

For $\zeta=1 / 10$, the diffusion coefficient exhibits a maximum in the region $0.820<\rho_{D_{\max }}<0.920$ and a minimum for $0.710<\rho_{D_{\min }}<0.780$, and temperatures between 0.350 and 0.450 . For densities lower than $\rho_{D_{\min }}$ diffusivity behaves normally, increasing as the density decreases. A similar behavior is verified for $\zeta=1 / 4$, with the diffusivity maximum located in the interval $0.870<\rho_{D_{\max }}<0.940$, and the minimum diffusivity in the range $0.740<\rho_{D_{\min }}<0.820$, for temperature interval ranging from 0.275 to 0.400 .

The loci of maxima and minima in diffusivity define a region of diffusion anomaly in the phase diagram, which is illustrated in Figs. 5(a) and 5(b), for $\zeta=1 / 10$ and $\zeta=1 / 4$ in the space of $\bar{T}$ versus $\rho$ and in Figs. 2(a) and 2(b) in the space of chemical potential $\bar{\mu}$. As can be seen, the maximum in diffusivity is located just above the critical line in the HDL phase, whereas the minimum in diffusivity is within the LDL, close to the gas-liquid coexistence line. Thus the diffusion anomalous region lies across the LDL-HDL critical 


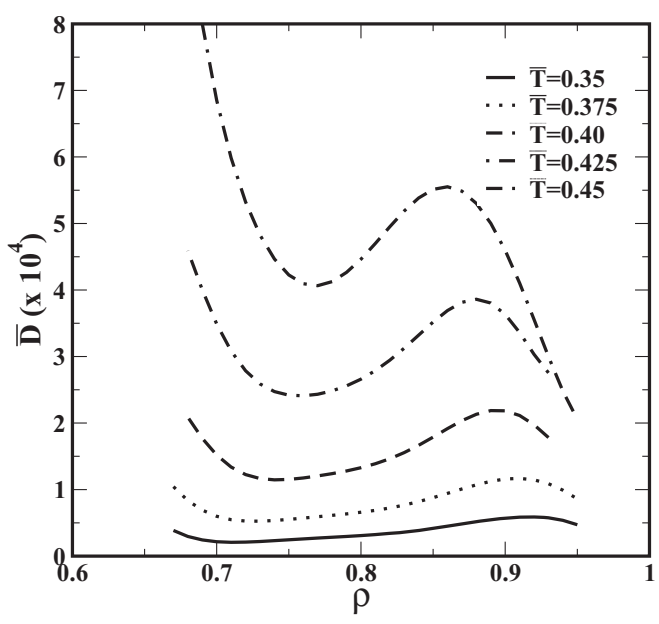

(a) $\zeta=1 / 10$

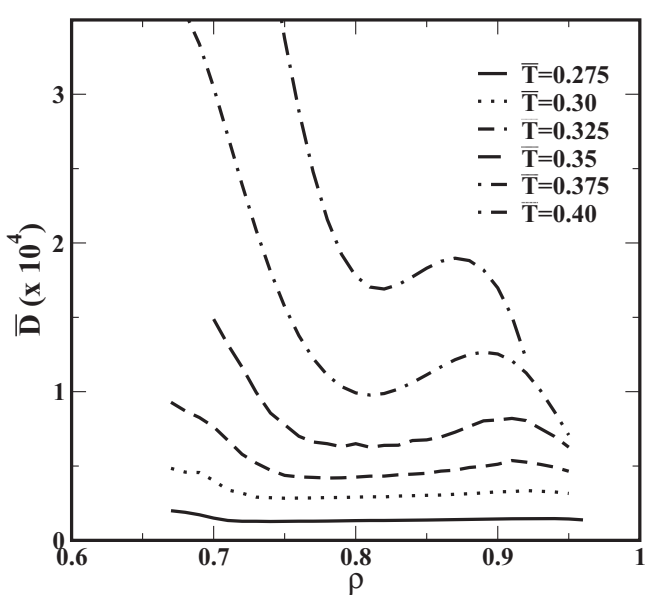

(b) $\zeta=1 / 4$

FIG. 4. Reduced diffusion constant as a function of density for (a) $\zeta=1 / 10$ for temperatures ranging from 0.350 to 0.450 and for (b) $\zeta=1 / 4$ for temperatures from 0.275 to 0.400 .

line. Since the LDL phase is characterized by bonds ordering, this explains the loss of particle mobility, as the LDL phase is approached from the HDL bond-disordered phase. Note that loss in mobility initiates in the bond-disordered phase close to the critical line, possibly related to large fluctuations in bonding density. On the other hand, inside the LDL phase, as density is further lowered, mobility again increases, in spite of bond order, probably due to the large increase in vacant sites, as the $2 / 3$ density of the fully translationally ordered phase is approached.

A point to note further is that the anomalous diffusion region is enveloped by the border of the region of density anomaly. This is different from the behavior presented by liquid water, but is common to other lattice models. ${ }^{15,16,27} \mathrm{~A}$ possible reason for this discrepancy is the fact that bonding is more rigid in the lattice model, thus reducing the mobility of particles as compared to continuous models, in which rotations allow for slightly distorted bonds.

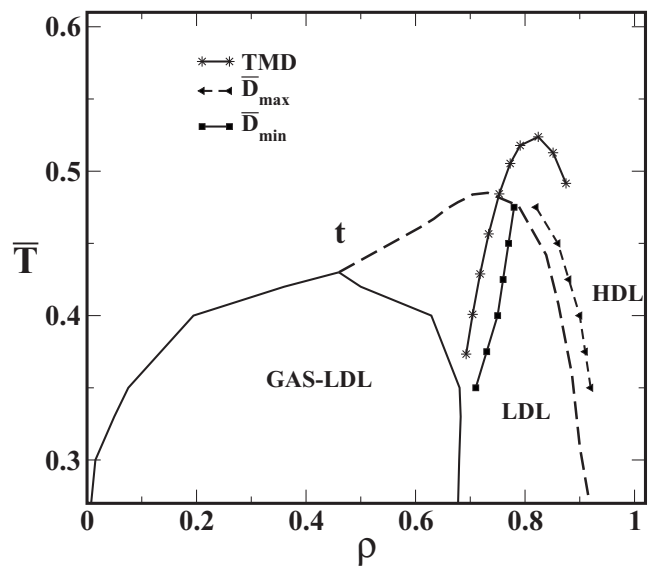

(a) $\zeta=1 / 10$

\section{B. Dynamic transitions}

In order to verify the existence of dynamic transitions and its possible relation to criticality, diffusivity was computed as a function of temperature, for fixed chemical potentials. The present analysis has been carried out in different regions of the phase diagram. As in the previous section, results for two different chemical potentials have been presented for both stronger and weaker bonding strength cases $(\zeta=1 / 10$ and $\zeta=1 / 4)$.

For $\zeta=1 / 10$, the behavior of the diffusion coefficient $\bar{D}$ with temperature was analyzed for chemical potentials $\bar{\mu}=$ -1.40 and $\bar{\mu}=-0.74$. The two values of $\bar{\mu}$ chosen are indicated by arrows in the phase diagram of Fig. 2(a). Figure 6(a) shows $\bar{D}$ versus $1 / \bar{T}$ for the two cases. For the lower chemical potential, the diffusion coefficient undergoes a dynamic transition at the critical line: At high temperatures, diffusivity follows non-Arrhenius polynomial behavior,

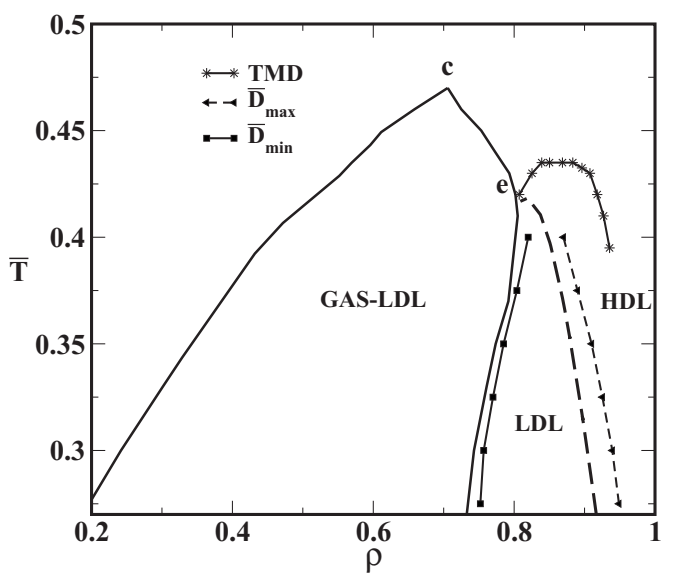

(b) $\zeta=1 / 4$

FIG. 5. Phase diagram $\bar{T}$ vs $\rho$ for (a) $\zeta=1 / 10$ and (b) $\zeta=1 / 4$. Continuous line corresponds to coexistence line and dashed line to critical line. For $\zeta=1 / 10$ continuous and critical lines meet at a tricritical point $t$, while for $\zeta=1 / 4$ continuous line ends in a critical point $c$ and critical line meet the continuous line at a critical end point $e$. Stars correspond to the density maxima, whereas squares and triangles denote the loci of diffusivity maxima and minima, respectively. 


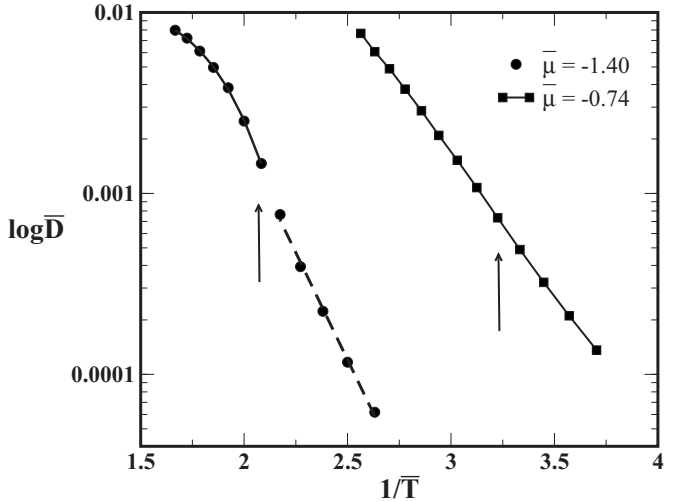

(a) $\zeta=1 / 10$

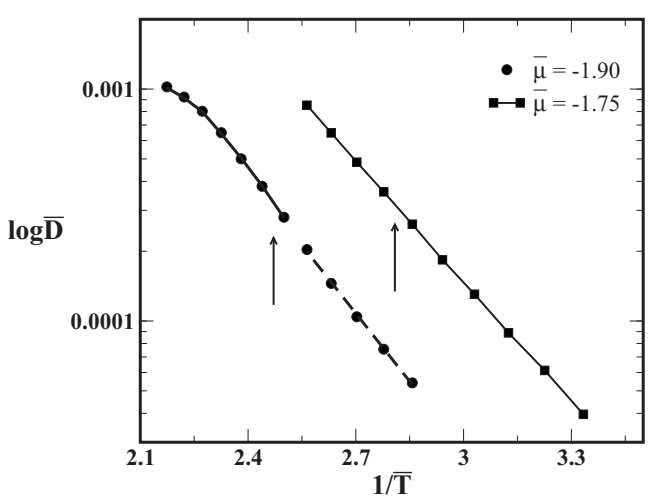

(b) $\zeta=1 / 4$

FIG. 6. Reduced diffusion constant vs inverse of reduced temperature for (a) $\zeta=1 / 10$ and (b) $\zeta=1 / 4$. For $\zeta=1 / 10$ diffusivity undergoes a dynamic transition for chemical potential $\bar{\mu}=-1.40$ as the critical line is crossed. Surprisingly, as the same critical line is crossed at higher chemical potential, $\bar{\mu}=-0.74$, diffusivity is not affected. For $\zeta=1 / 4$ diffusivity undergoes a dynamic transition for chemical potential $\bar{\mu}=-1.90$ at the critical line. Similar to the $\zeta=1 / 10$ system, diffusivity is no longer affected by crossing the critical line at a higher chemical potential $\bar{\mu}=-1.75$. Critical temperatures are indicated by arrows.

given generally by $\bar{D}=A_{0}+A_{1} \bar{T}+A_{2} \bar{T}^{2}+A_{3} \bar{T}^{3}$, which characterizes the system as a fragile liquid; in the low temperature region, diffusivity follows an Arrhenius law given by $\bar{D}$ $=B_{0} \exp \left(-B_{1} / \bar{T}\right)$, thus characterizing the system as a strong liquid. The coefficients $A_{i}$ and $B_{i}$ are fitting parameters, which are not investigated in this study. Surprisingly, for the higher chemical potential $\bar{\mu}=-0.74$ the dynamic crossover at the critical line is no longer detected. At this chemical potential, the critical line is crossed at a temperature $\bar{T} \approx 0.31$ $(1 / \bar{T} \approx 3.22)$ and, as can be seen in Fig. 6(a), the system dynamics is unaffected by the presence of the critical line.

For $\zeta=1 / 4$ the behavior of the diffusivity was also analyzed for two different chemical potentials, $\bar{\mu}=-1.90$ and $\bar{\mu}=-1.75$, as indicated by arrows in the phase diagram of Fig. 2(a). Results for the case of weaker hydrogen bonds are similar to the strong bonding case, as shown in Fig. 6(b). A dynamic transition is seen only for the lower chemical potential $\bar{\mu}=-1.90$, whereas for the higher chemical potential, $\bar{\mu}=-1.75$, diffusivity remains unaffected by the presence of the critical line, as in the previous case. Thus, despite the presence of a thermodynamic phase transition, a dynamic transition is absent, possibly implying that the structural change across the critical line is not significant, from the point of view of dynamics, at the higher densities.

A fragile-to-strong dynamic transition has been associated to a change of structure upon crossing the critical line. However, for higher chemical potentials the thermodynamic critical transition occurs within the diffusion anomalous region, as can be seen from Fig. 2(a). In this case the dynamics might be dominated by the anomalous behavior of the diffusion and not by the differences between the two structures on the two sides of the critical line.

\section{CONCLUSIONS}

In this paper we have addressed the question of the relation between critical lines and dynamic transitions. In order to highlight the answer to this question we have investigated the dynamic behavior of the BL water model. This model has been considered because it presents a single critical line that separates two fluid phases of different structures. Our study focuses on the diffusion anomaly and dynamic transitions, and on their relation to criticality.

In relation to the first feature, we have found that, similarly to other two length scales lattice models, the BL model presents a diffusion anomalous region inside the region of density anomalies. ${ }^{15,27}$

Second, we looked for dynamic transitions by analyzing the behavior of diffusivity with temperature across the critical line, at fixed chemical potentials. Our results showed that two different regimes may be found: If the critical line is crossed at low chemical potential, near the minimum in diffusivity, a fragile-to-strong transition is observed; for higher chemical potentials, near the diffusivity maximum, no dynamic transition is seen. Thus, different dynamic behavior is seen upon crossing distinct segments of the same critical line. Our explanation for this result is that the structural difference on both sides of the critical line, in the region of higher chemical potential, is not enough to provoke a change in diffusivity. In this particular region the diffusion anomaly dominates the dynamics.

In summary, our results indicate that dynamic transition and criticality are not directly associated. Instead, the fragileto-strong transition (and possibly strong-to-strong or even a fragile-to-fragile transitions) is the result of an expressive change in the structure of the liquid and of polymorphism. ${ }^{28}$

\section{ACKNOWLEDGMENTS}

We thank the financial support of the Brazilian science agencies CNPq and Capes. This work is partially supported by CNPq, INCT-FCx.

\footnotetext{
${ }^{1}$ M. Chaplin, See http://www.lsbu.ac.uk/water/anmlies.html for 63 anomalies of water.

${ }^{2}$ C. A. Angell, E. D. Finch, and P. Bach, J. Chem. Phys. 65, 3063 (1976). ${ }^{3}$ P. A. Netz, F. W. Starr, H. E. Stanley, and M. C. Barbosa, J. Chem. Phys. 115, 344 (2001).

${ }^{4}$ P. H. Poole, F. Sciortino, U. Essmann, and H. E. Stanley, Nature (London) 360, 324 (1992).

${ }^{5}$ O. Mishima and H. E. Satnley, Nature (London) 396, 329 (1998).

${ }^{6}$ O. Mishima, L. D. Calvert, and E. Whalley, Nature (London) 310, 393 (1984).
} 
${ }^{7}$ O. Mishima and Y. Suzuki, Nature (London) 419, 599 (2002).

${ }^{8}$ R. Martoňák, D. Donadio, and M. Parrinello, Phys. Rev. Lett. 92, 225702 (2004).

${ }^{9}$ R. S. Smith and B. D. Kay, Nature (London) 398, 302 (1999).

${ }^{10}$ A. Faraone, L. Liu, C.-Y. Mou, C.-W. Yen, and C. S.-H. Cen, J. Chem. Phys. 121, 10843 (2004).

${ }^{11}$ L. Liu, S.-H. Chen, A. Faraone, S.-W. Yen, and C.-Y. Mou, Phys. Rev. Lett. 95, 117802 (2005).

${ }^{12}$ L. Xu, P. Kumar, S. V. Buldyrev, S.-H. Chen, P. Poole, F. Sciortino, and H. E. Stanley, Proc. Natl. Acad. Sci. U.S.A. 102, 16558 (2005).

${ }^{13}$ C. A. Angell, J. Res. Natl. Inst. Stand. Technol. 102, 171 (1997).

${ }^{14}$ P. Kumar, G. Franzese, and H. E. Stanley, Phys. Rev. Lett. 100, 105701 (2008).

${ }^{15}$ M. M. Szortyka, M. Girardi, V. B. Henriques, and M. C. Barbosa, J. Chem. Phys. 130, 184902 (2009).

${ }^{16}$ M. Szortyka, M. Girardi, V. B. Henriques, and M. C. Barbosa, J. Chem. Phys. 132, 134904 (2010).
${ }^{17}$ G. M. Bell and D. A. Lavis, J. Phys. A 3, 568 (1970).

${ }^{18}$ C. Buzano, E. De Stefanis, and M. Pretti, J. Chem. Phys. 129, 024506 (2008).

${ }^{19}$ M. Pretti and C. Buzano, J. Chem. Phys. 121, 11856 (2004).

${ }^{20}$ C. Buzano, E. De Stefanis, A. Pelizzola, and M. Pretti, Phys. Rev. E 69, 061502 (2004).

${ }^{21}$ N. G. Almarza, J. A. Capitan, J. A. Cuesta, and E. Lomba, J. Chem. Phys. 131, 124506 (2009).

${ }^{22}$ D. A. Lavis, J. Phys. C 6, 1530 (1973).

${ }^{23}$ M. A. A. Barbosa and V. B. Henriques, Phys. Rev. E 77, 051204 (2008).

${ }^{24}$ A. P. Young and D. A. Lavis, J. Phys. A 12, 229 (1979).

${ }^{25}$ B. W. Southern and D. A. Lavis, J. Phys. A 13, 251 (1980).

${ }^{26}$ C. E. Fiore, M. M. Szortyka, M. C. Barbosa, and V. B. Henriques, J. Chem. Phys. 131, 164506 (2009).

${ }^{27}$ M. M. Szortyka, M. Girardi, and M. C. Barbosa, Physica A 380, 27 (2007).

${ }^{28}$ C. A. Angell, J. Phys.: Condens. Matter 19, 205112 (2007). 